\title{
Ultrasensitive and whole-course encapsulated field detection of 2019-nCoV gene applying exponential amplification from RNA combined with chemical probes
}

\author{
Simin Xia, Xi Chen
}

The HIT Center for Life Sciences, Harbin Institute of Technology, Harbin, P. R. China

KEYWORDS: 2019-nCoV, SARS-CoV-2, COVID-19, recombinase polymerase amplification, RNA virus, FRET, lateral flow

ABSTRACT: The newly identified 2019-nCoV (SARS-CoV-2) RNA virus has caused over 80,000 laboratoryconfirmed human infections in China (as of Mar. $1^{\text {st }}, 2020$ ), and is now becoming a global epidemic issue. As a result, highly sensitive, reliable and field-deployable methods to detect 2019-nCoV that can be developed in a shortest possible time are urgently needed. In this work, we introduce whole-course encapsulated and ultrasensitive field detection methods against 2019-nCoV gene. We applied isothermal exponential amplification methods via reverse transcription plus subsequent enzymatic recombinase amplification (ERA), a modified recombinase polymerase amplification (RPA), to amplify trace level of 2019-nCoV RNA. We designed both exo FRET probe and nfo affinity probe for easy detection of the amplified nucleic acids. These methods can be developed within a few days and as low as 0.32 aM (i.e. $0.32 \times 10^{-18} \mathrm{M}$ ) of RNA can be reliably detected.

Since the end of 2019, the word is experiencing an outbreak of a novel coronavirus called 2019-nCoV, or SARS-CoV-2, originally spreading in China, and is now causing a global epidemic issue ${ }^{1}$. 2019-nCoV is an RNA virus that causes acute pneumonia named COVID-19. The whole genome of 2019-nCoV was soon sequenced and released to public on Jan. $10^{\text {th }} 2020^{2}$, which served as the basis for nucleic acid-based diagnostics. Reverse transcription-polymerase chain reaction (RT-PCR) methods are among the initial detection approaches that meet the urgent need of 2019-nCoV viral detection ${ }^{3}$.

In the early stage of a viral epidemic explosion, local governments usually have not had taken strict control over it because the viral infection was still not yet prominent. Meanwhile, due to the lack of fielddeployable detection approaches, suspected infections have to go to centralized institutions or hospitals where advanced diagnostic methods are available; this could easily lead to latent cross transmission (Figure $1 \mathrm{~A}$ ) because human-to-human transmission had been revealed to occur among closely contacted persons ${ }^{4}$. Hence, sensitive and field detection methods against 2019-nCoV are urgently needed.

However, this is currently a challenging task. Reverse transcription-polymerase chain reaction (RT-PCR) is a powerful approach to detect RNA virus; yet it is not readily field-deployable because the high cost of real-time PCR machine and the expertise required to perform the analysis. Other detection methods such as antibody-based diagnostics are field-deployable but cannot be introduced in the early stage of a viral outbreak because of weeks or even months of time needed for immunization to produce high tilter antibodies; additionally, they are not as sensitive and specific as nucleic acid-based approaches ${ }^{5}$. Gene editing has recently been introduced as a novel field detection method against RNA ${ }^{6}$; yet it includes multiple reaction steps and its detection against 2019-nCoV has not been reported yet. 
In order to meet this urgent need, we herein introduced an ultrasensitive field-deployable exponential amplification approach to detect 2019-nCoV gene applying reverse transcription-enzymatic recombinase amplification (RT-ERA), combined with chemical probes (Figure 1B). ERA is a modified version of recombinase polymerase amplification (RPA ${ }^{7}$ from GenDx Biotech, belonging to isothermal nucleic acid amplification techniques ${ }^{8}$ that can be carried out at constant temperature without the need of thermo cycles. Additionally, we introduce a "whole-course encapsulated procedure for exponential amplification from RNA (WEPEAR)" for sample-in, results-out reliable detection of trace amount of viral RNA in one pot (Figure 1C). In the one-pot multi-enzyme reaction system, the RNase inhibitor (I) prevents the trace amount of RNA from degradation, reverse transcriptase (T) transcripts viral RNA to cDNA, recombinase $(\mathbf{R})$, polymerase $(\mathbf{P})$, single-stranded DNA binding protein $(\mathbf{B})$ and creatine kinase $(\mathbf{K})$ enable ERA reaction to proceed, and finally nuclease $(\mathbf{N})$ triggers the cleavage of chemical probes to give detectable readout (Figure 1B, C).

In order to establish a more quantitative RNA detection method, we prepared an RNA gene fragment of 2019-nCoV as the specimen. Within the entire genome of 2019-nCoV, we selected a part of " $N$ '-gene for detection (Figure S1A). We subsequently prepared a high-quality RNA fragment within " $\mathrm{N}$ "-gene via in vitro reverse transcription using T7 polymerase for following assay development (Figure S1B).

The first detection method we introduced is called exo FRET (fluorescence resonance energy transfer) probe for fluorogenic detection of 2019-nCoV gene (Figure 2). We designed an exo forward and reward primer pair for specific amplification of the RNA specimen via RT-ERA (Figure 2A). We performed BALST analysis to confirm that this primer pair indeed specifically detects 2019-nCoV virus but not any other species know so far. In addition, we designed an exo FRET probe that can be used to specifically detect the amplicon in the presence of exonuclease III (Exo III, Figure 2A). Exo FRET probe features 33bp bases at its $5^{\prime}$-side, $12 \mathrm{bp}$ bases at its $3^{\prime}$-side, a phosphate blocking group at its $3^{\prime}$-side so that this probe cannot act as a primer (Figure 2B). This FRET probe specifically hybridizes with one chain of the amplicon. Additionally, this probe features a 6-FAM modified T-residue (i6FAMdT) and a BHQ1 quencher modified T-residue (iBHQ1dT) in close proximity separated by 3 bases; thus, the fluorescence of FAM is temporally quenched by its FRET quencher-BHQ1. Finally, the exo FRET probe features an abasic tetrahydrofuran (THF) residue (idSp) between the FRET pair. THF residue is stable against Exo III when the FRET probe is in a single-stranded state, but will be cleaved by Exo III when the probe hybridizes with one chain of the amplicon in a double-stranded state. Then BHQ1 releases and the fluorescence of FAM moiety is restored. We found that 100 unites of Exo III serve as an optimal level to trigger the fluorescence increase (Figure S2).

We have also introduced a WEPEAR procedure for sample-in, results-out one-pot detection of RNA in a sensitive and reliable way. While using ERA to detect DNA in one-pot fashion has been described before, fully enclosed one-pot detection of RNA using RT-ERA in which RT is first conducted separately without being interfered by ERA has not been explicitly described. Although RT-ERA or RT-RPA can still work when both RT and ERA/RPA are conducted simultaneously in one pot, optimal results will be obtained if RT step is performed first without being interfered by ERA, followed by ERA reaction; in addition, RT and ERA usually have different optimal reaction temperatures (RT: $37{ }^{\circ} \mathrm{C}$, ERA: $40{ }^{\circ} \mathrm{C}$ ). In this WEPEAR protocol, RNA sample is added into a reaction mixture containing all necessary components for both RT and ERA aside from ERA activator- $\mathrm{Mg}^{2+}$, while $2 \mu \mathrm{lof} \mathrm{Mg}^{2+}$ is loaded inside the lid of the reaction vial. $\mathrm{Mg}^{2+}$ liquid will still stay inside the lid after gently close the lid due to surface tension. The reaction vial is 
first placed in a $37{ }^{\circ} \mathrm{C}$ water bath for $60 \mathrm{~s}$ to allow only RT to take place. After spin, $\mathrm{Mg}^{2+}$ activator is mixed and triggers the ERA reaction. The reaction vial is heated to $40{ }^{\circ} \mathrm{C}$ for 4 min to allow initial RPA reaction to proceed. Finally, shake and spin the vial again (essential to enhance detection sensitivity since the reaction mixture is highly viscous), and let the reaction to proceed for additional 26 min at 40 ${ }^{\circ} \mathrm{C}$. Finally, the reaction product can be simply detected via green fluorescence excited by blue light. During the entire course, the reaction proceeds through four steps, RT, ERA-activation, pre-reaction, and final reaction, to give fluorescence readout without uncover the lid at all, hence greatly reduces any possible contaminations (e.g. via aerosol) and ensures the reliability of this approach to detect trace amount of RNA.

We were happy to find that this exo FRET probe in combination with WEPEAR gives consistently ultrahigh sensitivity. As can been seen from Figure 2D, as low as 1 ag $\left(10^{-18} \mathrm{~g}\right)$ of RNA sample can be detected when compared to negative control $(0 \mathrm{ag})$. The fluorescence intensity of each reaction solution increases along with increased mass of RNA and the fluorescence intensity reaches maximal when above 1 pg of RNA was added. We also used fluorescence spectrometer to quantitatively analyze the fluorescence enhancement and further confirmed that the detection limit of this approach is between $10^{-2}$ to 1 ag (Figure 2E). In account of the M.W. of the RNA (147655 Da, $21 \mu$ l of sample), lower than $0.32 \mathrm{aM}$ (i.e. $<0.2$ copy/ $\mu$ ) of RNA could be detected suggesting that this is an ultrasensitive detection method. We also plotted the fluorescence intensity of each reaction solution against the mass of RNA and found that a half effect mass of RNA sample to be detected is around $8 \mathrm{fg}$ (Figure S3). Since this method only requires a mimi-blue light imaging plate, or simply a mini-blue light flashlight (few dollars) plus an orange colored plastic film, this detection method is field-deployable and can be applied to grass-roots clinics. Meanwhile, we demonstrated that this exo FRET probe can also be implemented in an advanced real-time PCR station that allows real-time detection of the fluorescence enhancement (Figure S4). The maximal reaction intensity is reached after $25 \mathrm{~min}$, suggesting that $30 \mathrm{~min}$ of total reaction time in our protocol is a suitable parameter using exo FRET probe.

Using exo FRET probe, some setups are still needed such as a blue-light imaging plate and a temperature-controlled heating block. In order to make the detection approach simpler, or even can be conducted at home, we were motivated to design a so-called nfo affinity probe system using lateral flow (LF) strips for detection (Figure 3). We employed the nfo forward primer (same as the exo forward primer) and nfo reward primer (same sequence as the exo forward primer but features a 5'-biotin affinity tag) that can be paired to amplify amplicon within " $\mathrm{N}$ '-gene. In addition, we designed a nfo affinity probe containing $33 \mathrm{bp}$ in its $5^{\prime}$-side and $15 \mathrm{bp}$ in its $3^{\prime}$-side that can hybridize with the biotintagged chain of the amplicon, a 5-FAM moiety (serves as an antigen rather than a fluorophore), a THF site that can be potentially cleaved by Endonuclease IV (Endo IV), and a 3'-phosphate blocking group so that this probe cannot act as a primer. Endo IV cannot cleave the THF site of the probe, but can cleave the THF site when the probe hybridizes with the biotin-tagged amplicon chain in a double-stranded state. After cleavage of the THF site, the 3 '-side of the probe is released, converts the nfo probe to a primer that initializes polymerization in the RT-ERA reaction. In the end a FAM and a biotin dual-tagged amplicon is generated (Figure 3B, down), which could be easily detected using a lateral flow strip without using any particular setups.

The lateral flow strip features a testing line that is immobilized with $\alpha$-biotin antibody and a control line that is immobilized with an antibody against $\alpha$-FAM antibody (i.e. $\alpha$-[ $\alpha$-FAM-gold] antibody). In 
addition, there is a colloidal gold particle region (the gold particle is coated with $\alpha$-FAM antibody) below the two lines and above the sample pad. When the sample pad of the test strip is inserted into a RT-ERA reaction solution, the colloidal gold particle will flow along the eluent and cross test and control lines. If the bis-tagged amplicon is presented in the RT-ERA reaction, it will bridge the gold particle and the $\alpha$ biotin antibody in the test line, turns the test line to red (Figure $3 C$, right). Meanwhile the control line that contains $\alpha$-[ $\alpha$-FAM-gold] antibody shall bind the $\alpha$-FAM antibody-coated gold particle, suggesting that the LT strip is effective.

Using the WEPEAR protocol introduced above, we performed the RT-ERA reaction in the presence of different amount of RNA, from extremely low level of $10^{-2}$ ag (i.e. $10^{-20} \mathrm{~g}$ ) to $10 \mathrm{ng}$. We were delighted to find that this detection approach is as sensitive (or even more) as the above-mentioned exo FRET probe system and that as low as 1 ag of RNA can be clearly detected visually when compared to the blank control (Figure 3D). Sometimes blank control reaction may also give a faint but visually detectable test line; hence it is necessary to always include a blank control for each time of detection, and read the test line of both the sample and the blank control strips at the same time within the first 2-3 min.

In this nfo affinity detection approach, temperature-controlled water bath or heating block may still be needed. These setups are usually inexpensive, but may not be always available. Therefore, we checked that if nfo affinity detection approach could be performed simply using a thermos cup that is easily available. By gradually adding hot water into a room temperature water inside a thermos cup with stirring, it is easier than expected to make $40{ }^{\circ} \mathrm{C}$ water. Then a control reaction vial ( $0 \mathrm{pg}$ RNA) and a reaction vial (100 pg RNA) were put into the cup via a float and then cover the thermos cup by a foam (Figure $3 \mathrm{E}$ ). The temperature can be easily maintained without over $1^{\circ} \mathrm{C}$ of drop after $30 \mathrm{~min}$. In fact, for multiple tests in our hand, no single case has had ever occurred when the temperature drop reached 2 ${ }^{\circ} \mathrm{C}$ in $30 \mathrm{~min}$. The RT-ERA reaction mixture was diluted and tested using gold LF strips and we found that 2019-nCoV RNA could be easily detected using this household setup (Figure 3F). Usually an RNA purification step of clinic sample is needed for the optimal testing sensitivity and specificity. Motivated by the ultrahigh sensitivity of this nfo affinity detection approach, we mixed 2019-nCoV RNA gene directly with diluted throat swab without extra sample processing nor RNA purification. Delightfully, 2019-nCoV RNA in throat swap could still be detected clearly compared to blank control, suggesting that even sample processing step could be skipped using this ultrasensitive nfo affinity detection approach (Figure 3G). These experiments highlighted that nfo affinity probe could be applied not only in a fielddeployable way, but also in a household fashion.

In summary, in order to meet the urgent requirement for sensitive and field detection of 2019-nCoV, we herein introduced ultrasensitive field detection approaches against 2019-nCoV RNA gene. The lower detection limit can reach below $1 \mathrm{ag}(0.32 \mathrm{aM}$, or $0.2 \mathrm{copy} / \mu \mathrm{l})$, which is among the highest sensitive detection approaches at the moment ${ }^{9}$. More importantly, these nucleic acid detection approaches are field-deployable or even household. The exo FRET probe detects the RNA gene via fluorescence enhancement while the nfo affinity probe detects the RNA gene using LF strips. Furthermore, these detection approaches could be developed in a shortest possible time, within few weeks or even few days once the viral genome information is available, thus fully meet the requirements of field detection when a viral outbreak occurs. We hope that the exo FRET probe, nfo affinity probe and the respective primers we designed here could facilitate the rapid, sensitive and field detection of 2019-nCoV infected patients. 


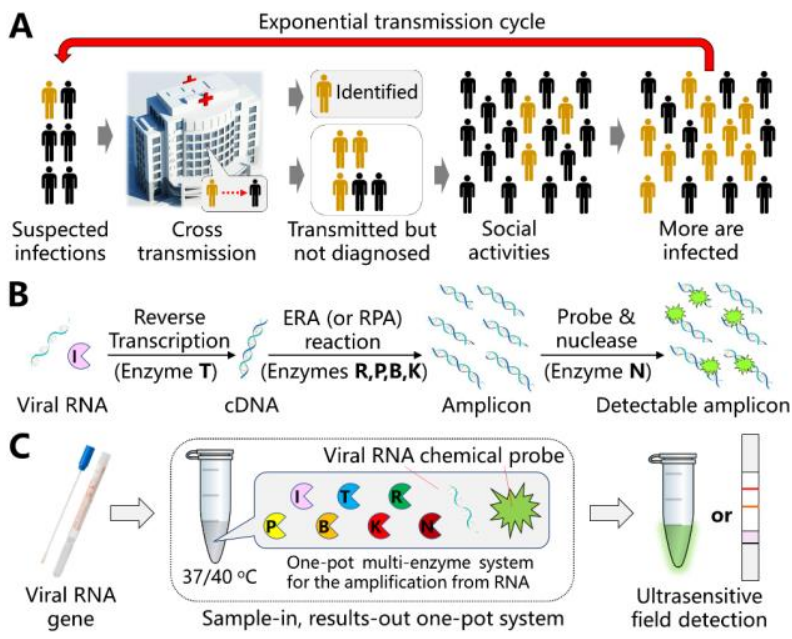

Figure 1. A. Motivation of this study and the general schematic view of the detection approaches. A, Due to crosstransmission of 2019-nCoV, sensitive and field-deployable nucleic acid detection methods are urgently needed. B. Schematic view of the detection steps. C. Sample-in, results-out one-pot field detection in this study.

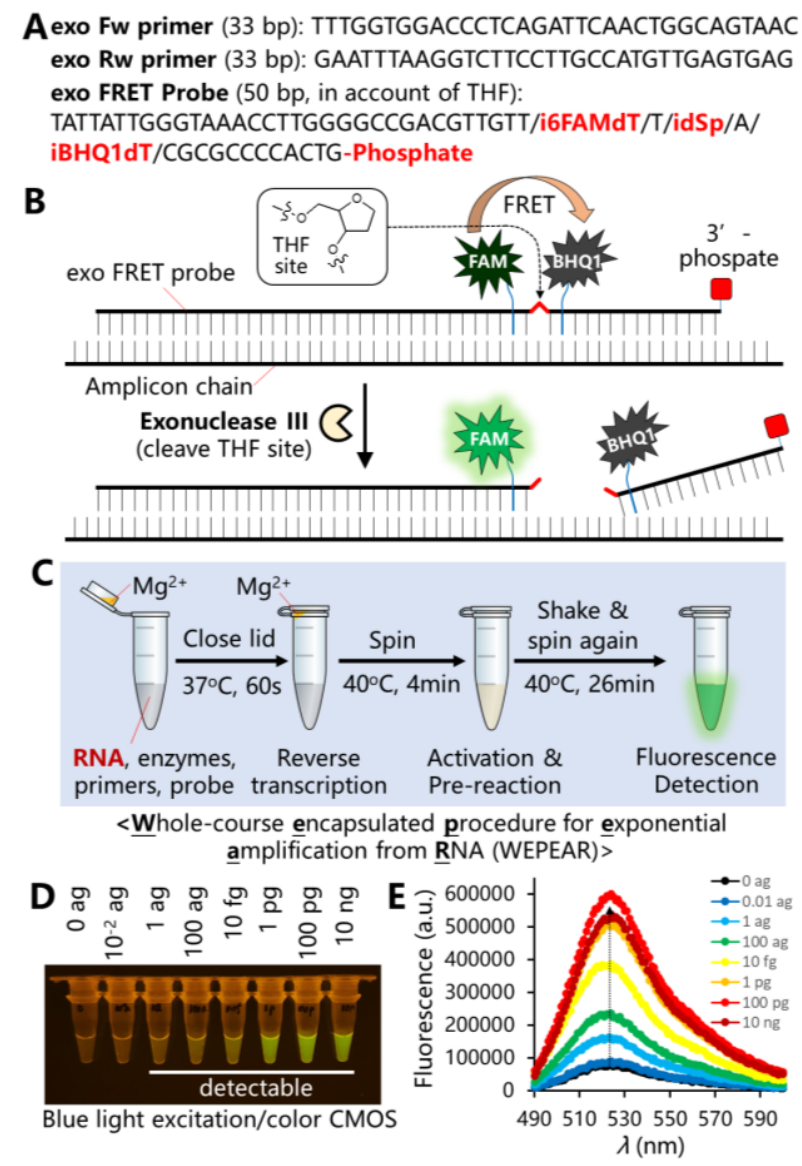

Figure 2. Ultrasensitive and field-deployable WEPEAR approach to detect 2019-nCoV gene using exo FRET probe. A, the sequence and structural information of the exo forward primer, exo reward primer and exo FRET probe. $B$, the principle of exo FRET probe detection. C, Schematic view of the WEPEAR protocol. D, Fluorescence detection using a blue light plate. $E$, quantitative analysis of the fluorescence enhancement. 
A nfo Fw primer (33 bp): TTTGGTGGACCCTCAGATTCAACTGGCAGTAAC

nfo Rw primer (33 bp): Biotin-GAATTTAAGGTCTTCCTTGCCATGTTGAGTGAG nfo affinity probe ( $49 \mathrm{bp}$, in account of THF):

GCGATCAAAACAACGTCGGCCCCAAGGTTTACC/idSp/AATAATACTGCGTCT-Phosphate
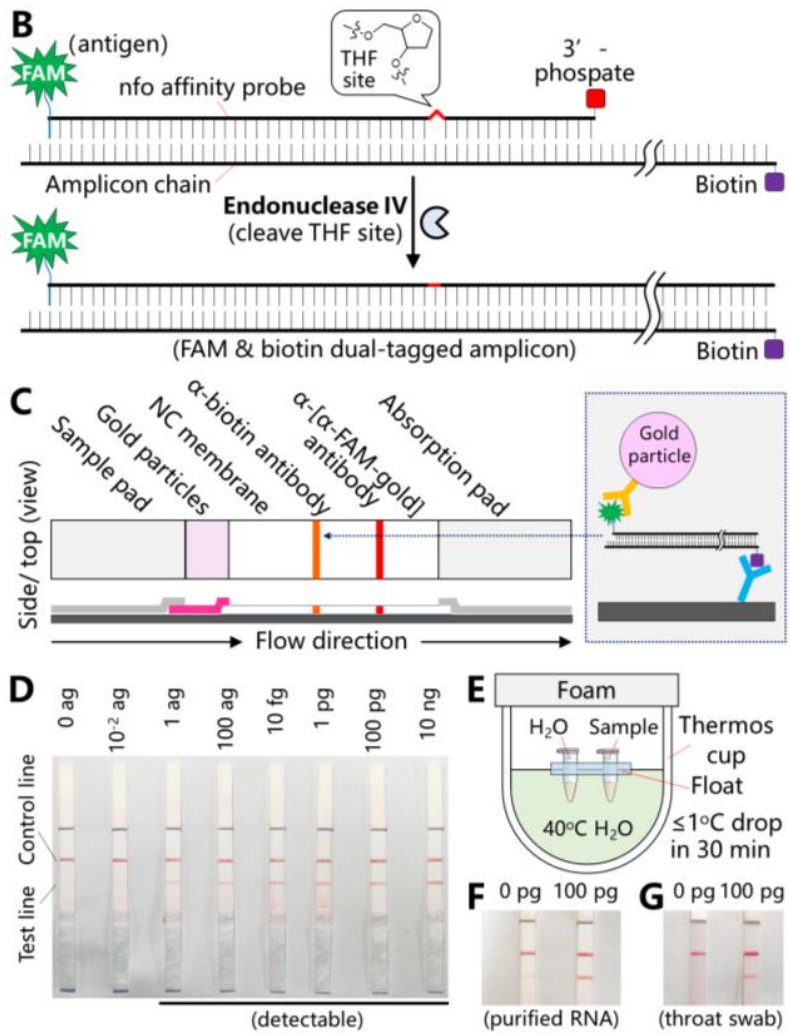

Figure 3. Ultrasensitive and household detection of 2019-nCoV gene using the nfo affinity probe plus LF strips. A, sequence and structural information of nfo forward prime, nfo reward primer and nfo affinity probe. B, the principle of nfo affinity probe detection. C, schematic view of the principle of LF strips detection. D. Readouts of LF strips. E, a thermos cup can be used as the primary setup for RT-ERA reaction. F, results of the LF strips using a thermos cup setup. G. RNA specimen in throat swab can be directly used in the detection.

\section{ASSOCIATED CONTENT}

Supporting Information. 2019-nCoV "N"-gene sequence, T7 transcription protocol, RT-ERA protocols, detection using lateral flow strips, and other relevant information are available free of charge via the internet at http://pubs.acs.org."

\section{AUTHOR INFORMATION}

\section{Corresponding Author}

*chenxihit@hit.edu.cn

\section{Author Contributions}

The manuscript was written through contributions of all authors.

\section{ACKNOWLEDGMENT}


We thank the core facilities at The HIT Center for Life Sciences of Harbin Institute of Technology (HIT) and the funding support from HIT.

\section{ABBREVIATIONS}

CDNA, complementary DNA; COVID-19, coronavirus disease 2019; RPA, recombinase polymerase amplification; ERA, enzymatic recombinase amplification; LF, lateral flow; NC, nitrocellulose; RT, reverse transcription; PCR, polymerase chain reaction; WEPEAR, whole-course encapsulated exponential amplification from RNA; 6-FAM, 6-carboxyl-fluorescein; BHQ1, black hole quencher 1.

1. Wang, C.; Horby, P. W.; Hayden, F. G.; Gao, G. F., A novel coronavirus outbreak of global health concern. Lancet 2020, 395 (10223), 496-496.

$2 . \quad$ Zhang, Y.-Z., Virological.org 2020.

3. (a) Corman, V. M.; Landt, O.; Kaiser, M.; Molenkamp, R.; Meijer, A.; Chu, D. K. W.; Bleicker, T.; Brunink, S.; Schneider, J.; Schmidt, M. L.; Mulders, D. G. J. C.; Haagmans, B. L.; van der Veer, B.; van den Brink, S.; Wijsman, L.; Goderski, G.; Romette, J. L.; Ellis, J.; Zambon, M.; Peiris, M.; Goossens, H.; Reusken, C.; Koopmans, M. P. G.; Drosten, C., Detection of 2019 novel coronavirus (2019-nCoV) by real-time RT-PCR. Eurosurveillance 2020, 25 (3), 23-30; (b) Chu, D. K. W.; Pan, Y.; Cheng, S. M. S.; Hui, K. P. Y.; Krishnan, P.; Liu, Y.; Ng, D. Y. M.; Wan, C. K. C.; Yang, P.; Wang, Q.; Peiris, M.; Poon, L. L. M., Molecular Diagnosis of a Novel Coronavirus (2019-nCoV) Causing an Outbreak of Pneumonia. Clin Chem 2020.

4. (a) Huang, C.; Wang, Y.; Li, X., Clinical features of patients infected with 2019 novel coronavirus in Wuhan, China. Lancet 2020, 395 (10223), 496-496; (b) Li, Q.; Guan, X.; Wu, P.; Wang, X.; Zhou, L.; Tong, Y.; Ren, R.; Leung, K. S. M.; Lau, E. H. Y.; Wong, J. Y.; Xing, X.; Xiang, N.; Wu, Y.; Li, C.; Chen, Q.; Li, D.; Liu, T.; Zhao, J.; Li, M.; Tu, W.; Chen, C.; Jin, L.; Yang, R.; Wang, Q.; Zhou, S.; Wang, R.; Liu, H.; Luo, Y.; Liu, Y.; Shao, G.; Li, H.; Tao, Z.; Yang, Y.; Deng, Z.; Liu, B.; Ma, Z.; Zhang, Y.; Shi, G.; Lam, T. T. Y.; Wu, J. T. K.; Gao, G. F.; Cowling, B. J.; Yang, B.; Leung, G. M.; Feng, Z., Early Transmission Dynamics in Wuhan, China, of Novel Coronavirus-Infected Pneumonia. N Engl J Med 2020.

5. $\quad$ Balmaseda, A.; Stettler, K.; Medialdea-Carrera, R.; Collado, D.; Jin, X.; Zambrana, J. V.; Jaconi, S.; Cameroni, E.; Saborio, S.; Rovida, F.; Percivalle, E.; Ijaz, S.; Dicks, S.; Ushiro-Lumb, I.; Barzon, L.; Siqueira, P.; Brown, D. W. G.; Baldanti, F.; Tedder, R.; Zambon, M.; de Filippis, A. M. B.; Harris, E.; Corti, D., Antibody-based assay discriminates Zika virus infection from other flaviviruses. Proc Natl Acad Sci U S A 2017, 114 (31), 8384-8389.

6. $\quad$ Myhrvold, C.; Freije, C. A.; Gootenberg, J. S.; Abudayyeh, O. O.; Metsky, H. C.; Durbin, A. F.; Kellner, M. J.; Tan, A. L.; Paul, L. M.; Parham, L. A.; Garcia, K. F.; Barnes, K. G.; Chak, B.; Mondini, A.; Nogueira, M. L.; Isern, S.; Michael, S. F.; Lorenzana, I.; Yozwiak, N. L.; MacInnis, B. L.; Bosch, I.; Gehrke, L.; Zhang, F.; Sabeti, P. C., Field-deployable viral diagnostics using CRISPR-Cas13. Science 2018, 360 (6387), 444-448.

7. $\quad$ Piepenburg, O.; Williams, C. H.; Stemple, D. L.; Armes, N. A., DNA detection using recombination proteins. Plos Biol 2006, 4 (7), 1115-1121.

8. (a) Zhao, Y. X.; Chen, F.; Li, Q.; Wang, L. H.; Fan, C. H., Isothermal Amplification of Nucleic Acids. Chem Rev 2015, 115 (22), 12491-12545; (b) Daher, R. K.; Stewart, G.; Boissinot, M.; Bergeron, M. G., Recombinase Polymerase Amplification for Diagnostic Applications. Clin Chem 2016, 62 (7), 947-958.

9. Obande, G. A.; Singh, K. K. B., Current and Future Perspectives on Isothermal Nucleic Acid Amplification Technologies for Diagnosing Infections. Infect Drug Resist 2020, 13, 455-483. 